\title{
Author Correction: Therapeutic vaccination against leukaemia via the sustained release of co-encapsulated anti-PD-1 and a leukaemia-associated antigen
}

Xiaoling Xie D, Yuxing Hu, Tong Ye Di , Yiran Chen, Lijuan Zhou, Feng Li, Xiaobo Xi, Shuang Wang, Yanjie He, Xiaoyong Gao (D), Wei Wei(D), Guanghui Ma(D) and Yuhua Li (D)

Correction to: Nature Biomedical Engineering https://doi.org/10.1038/s41551-020-00624-6, published online 12 October 2020.

In the version of this Article originally published, the labels ' $\mathrm{M}(\mathrm{aP})$ ' and ' $\mathrm{aP}$ ' in the legend for Fig. 4a were associated with the wrong curves; 'M(aP)' refers to the red curve and 'aP' to the blue curve. Furthermore, in the legend for Fig. 5c, the label 'M(pE/aP)' was missing the bracket on the right. All versions of the Article have been amended.

Published online: 19 April 2021

https://doi.org/10.1038/s41551-021-00726-9

๑ The Author(s), under exclusive licence to Springer Nature Limited 2021 